\title{
LE PRIME DISPUTE TRA ARCHITETTI LOMBARDI E FORESTIERI NEL CANTIERE DEL DUOMO
}

\author{
LUCIANO PATETTA (*)
}

Riassunto. - Dai Libri Mandatorum (poi trascritti negli Annali della Fabbrica del Duomo, Milano 1877-1885) abbiamo una grande quantità di notizie sui magistri e sugli inzignierii lombardi, francesi e tedeschi chiamati, alla fine del Trecento, da Galeazzo Visconti e dai Deputati cittadini a progettare una cattedrale gotica, degna di quelle europee. Le discussioni e le dispute hanno riguardato la planimetria del Duomo e gli schemi della sua sezione, per una scelta della tradizione locale o di una imitazione delle grandi cattedrali di Francia. Soprattutto con l'arrivo da Parigi dell'architetto Jean Mignot (1400 ca.) si scontrarono due teorie che si fondavano, rispettivamente, una sui principi della geometria e della matematica, l'altra sulle esperienze costruttive delle maestranze con l'uso dei materiali locali. Le polemiche si fondavano su due affermazioni contradditorie, rimaste poi emblematiche: ars sine scientia nibil est e scientia sine arte nibil est.

$* * *$

ABSTRACT. - From the Libri Mandatorum (later on in the Annali della Fabbrica del Duomo, Milan 1877-85) we have a great quantity of news about the magistri of yard and about Lombard, French and German inzignierii, called at the end of Fourteenth Century, by Gian Galeazzo Visconti and by the Delegates of the town to built a gothic cathedral, whorty of whose of Europe. The discussions and the disputations were about the plan of the Duomo and the pattern of his section, for the choise of local tradition or imitation of great French cathedrals. Above all the arrival from Paris of the architect Jean Mignot (approximately 1400) clashed two theories well-grounded, one on principles of the geometry and the mathematic, and the other on the building experience of the workers on the use of local building materials. The controversies founded on two opposite assertions: ars sine scientia nibil est and scientia sine arte nibil est.

(*) Politecnico di Milano. 
Le dimensioni enormi del Duomo (opus amplissimum et quidam templum immensum) corrispondevano alle ambizioni di Antonio da Saluzzo e di suo cugino Gian Galeazzo Visconti, conte di Virtù e ancora vicario imperiale, nelle cui mire politiche e militari rientravano i legami matrimoniali con le Case regnanti europee e un vasto programma edilizio (il potenziamento dei cantieri dei castelli di Pavia e di Milano, del Duomo di Como e di quello di Monza, delle chiese del Carmine a Pavia e Milano, ed infine della Certosa di Pavia). Ma le enormi proporzioni del Duomo corrispondevano anche alla concezione di un tempio che potesse accogliere - come Arca della Salvezza - tutti i cittadini, secondo l'esempio delle cattedrali d'Oltralpe edificate negli ultimi duecento anni, che potevano accogliere tutti i fedeli di città che peraltro contavano solo alcune decine di migliaia di abitanti. Confermavano l'importanza, anche simbolica, che Gian Galeazzo Visconti (primo duca di Milano nel 1395) dava all'edificazione di una grande cattedrale, l'assegnazione alla Fabbrica del Duomo del diritto di estrarre il Serizzo da cave di proprietà della città e dalle sue cave di marmo di Candoglia, nonché alcune esenzioni doganali.

Poco sappiamo dei primi lavori nel cantiere del Duomo tra il 1386 (convenzionale data di fondazione) e il 1390, anni in cui si stabilirono i ruoli dei Deputati cittadini (all'inizio in numero di 19), della Corte e della Curia, si stabilirono i regolamenti amministrativi ed esecutivi della Fabbrica, i diritti e i doveri dei geometri, matematici, maestri di muro, lapicidi, carpentieri, vetrai ecc. Si cominciò subito a documentare le forniture, i lavori, le decisioni, le spese e persino le disputationes (tipiche anche degli ambienti filosofici e teologici medievali) nei Libri Mandatorum e in "libri mastri" di controllo (trascritti poi negli Annali della Fabbrica, Milano 1877-85). Purtroppo sono andati perduti i primi Libri relativi agli anni 1387-1389.

La prima figura importante di magister del cantiere, probabilmente con le responsabilità maggiori, di cui abbiamo notizie è quella di Simone da Orsenigo (dal 1387 al 1391 inzignerio), ma erano presenti anche Giacomo da Verona (dal 1388) alcuni Maestri Campionesi (tra cui forse già Giacomo da Campione e Marco da Carona chiamato nei documenti de Frixono, che nel 1388 fece un modello in legno per i piloni, e Bonino da Campione) Giovannino de' Grassi (dal 1389) Lorenzo degli Spazi (dal 1389), nonché due forestieri, Nicolas de Bonaventure (altrimenti detto Bonaventis) e Anechino de Alemagna (dagli anni 1387-89). ${ }^{1}$ La presenza di questi due magistri (architetti?) tardogotici d'Oltralpe è la prima testimonianza delle scelte culturali della 
Corte viscontea, nella quale contavano da tempo i gusti anche decorativi del cosiddetto "gotico internazionale" coltivati dalla sposa di Gian Galeazzo, Isabella di Valois, figlia del re di Francia, e dei suoi consiglieri francesi, nonché i rapporti politico-commerciali che molti autorevoli milanesi, vicini ai Visconti, tenevano con i centri dell'Inghilterra, della Francia e della Germania dell'imperatore Venceslao. L'insieme di questi fattori è stato alla base della scelta di abbandonare - almeno in parte - la grande tradizione milanese del Romanico e del Trecento lombardo, ed anche di trascurare gli importanti esempi recenti di cattedrali italiane, come S. Maria del Fiore a Firenze, il duomo di Orvieto e quello di Siena, per adottare alcuni caratteri strutturali e decorativi del gotico europeo.

E' probabile, comunque, che nei primi anni del cantiere l'attività dei due forestieri, il francese e il tedesco, sia stata prevalentemente di carattere artistico e decorativo (salvo l'impostazione dei tre finestroni ogivali dell'abside semiottagonale, per i quali si è fatto il nome di Simone de Bonaventure). Infatti, il Duomo ha avuto, senz'altro attraverso modelli lignei e schemi grafici, un'impostazione generale di stampo sostanzialmente italiano, con aspetti addirittura lombardi, proprio quell'impianto marcatamente tradizionale sugli sviluppi del quale si concentreranno, come vedremo, le critiche degli architetti tedeschi e francesi giunti a Milano nel decennio tra il 1391 e il 1401. La planimetria del Duomo risultava compatta anziché articolata, era assente il deambulatorio con le relative cappelle radiali attorno al presbiterio, presentava il transetto aggettante e dunque confermava la pianta "a croce latina", aveva muri perimetrali massicci (anziché "smaterializzati" dalle vetrate) con contrafforti aggettanti (collegati da una fondazione continua), che escludevano la resistenza a future spinte agenti verso l'esterno, e quindi, esplicitamente, escludevano un futuro sistema di archi rampanti (gli archi rampanti che verranno eseguiti nel Duomo non avranno "funzione spingente" sulle reni delle arcate trasversali, ma quasi solamente un carattere decorativo) (Fig. 1).

Infine, era prevista l'edificazione, all'incrocio tra il capocroce e il transetto, di un tiburio, secondo la secolare tradizione lombarda e del

1 Cfr. T. Zerbi, L'ordinamento aziendale della "Fabbrica" del Duomo di Milano secondo i "Capitoli" del 1387, in Atti del congresso internazionale sul Duomo di Milano, Milano 1969, I, pp.53-60. 
gotico cistercense dell'abbazia di Chiaravalle, ${ }^{2}$ e dunque uno sviluppo in altezza in questo punto dell'organismo e non in facciata, con le torri, come in tutte le cattedrali gotiche. Già nel 1387 fu fatto per il tiburio un modello, tiburium unum pombli, forse eseguito da Anechino de Alemagna. ${ }^{3}$
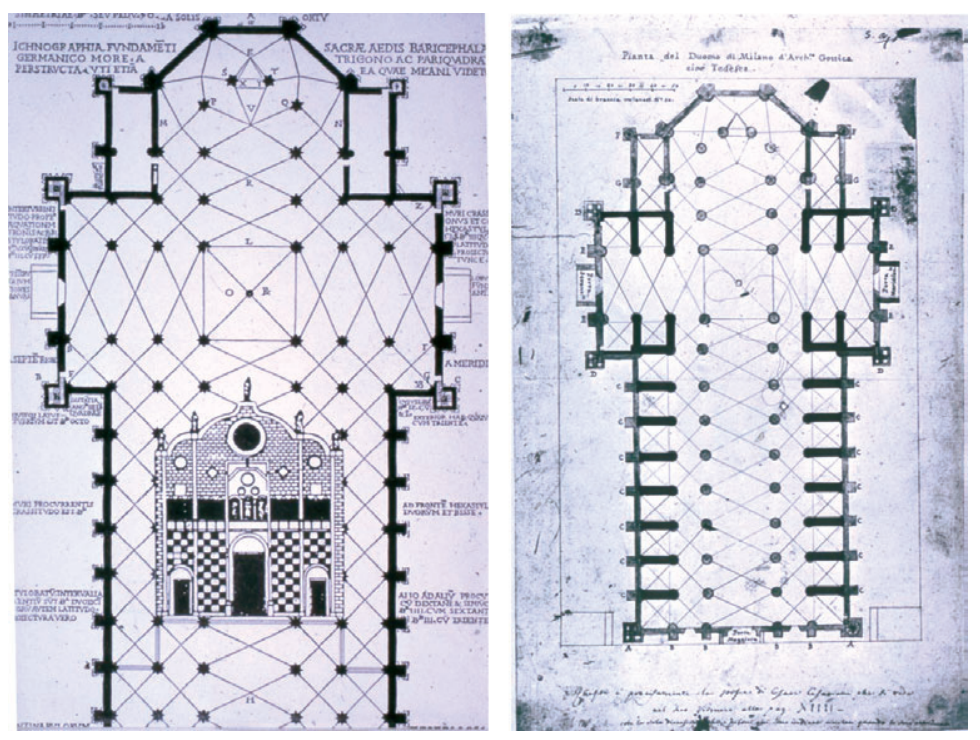

Fig. 1. A sinistra: Pianta del Duomo, con sovrapposta la facciata di S. Maria Maggiore, che arrivava fino alla quinta campata, ancora in piedi nel 1489 (da C. Cesariano, 1521, Libro $1^{\circ}$ ). A destra: Pianta del Duomo con indicata la proposta delle cappelle laterali (1400), riportate sul foglio Raccolta Bianconi, II, p.1, $f$ B.

Proprio le critiche degli architetti forestieri a questo impianto di tradizione italica e alle prime strutture eseguite (l'abside, le due sagrestie e alcune campate dei muri perimetrali) e gli argomenti delle loro dispute con le maestranze locali hanno finito per dar vita a una "questione del Duomo" di contenuto specificatamente costruttivo, quasi che il problema di una cattedrale gotica fosse solo di statica e di schemi strutturali,

2 Cfr. L. Patetta, Permanenze ed evoluzione del tiburio lombardo in M.C. Loi, L. Patetta (a cura di), Tradizioni e regionalismi nel primo Rinascimento italiano, Milano 2005, pp.35 e seg. pendice 14 .

Cfr. Annali della Veneranda Fabbrica del Duomo, Milano 1877-85, I², Ap- 
di cimento ed equilibrio tecnologico, anziché un insieme di molteplici valenze e problematiche, tra cui lo sterminato repertorio delle sculture, lo straordinario programma decorativo, ma sopratutto un'immagine e una rappresentazione simbolica con implicazioni teologiche e metafisiche.

Voglio accennare a quest'ultimo aspetto, prima di ripercorrere gli sviluppi di un dibattito sulle difformità del Duomo rispetto a una corretta goticità, argomento sul quale prevalentemente si sono concentrati alcuni dei più noti studi storici, ${ }^{4}$ interessati a trovare conferme razionalistiche $\mathrm{e}$ prove di una supposta metodologia progettuale scientifica del gotico.

E' riscontrabile nel Duomo (anche se non esplicitamente descritta nei documenti dell'epoca) una rappresentazione dell'universo, una imago mundi, un'immagine simbolica del cosmo come rivelato nelle Sacre Scritture, una sintesi mistica e reale della Gerusalemme Celeste. ${ }^{5}$ Simboli suggeriti certamente da dottori di astronomia e da teologi riguardavano la chiesa come un'immagine del cielo, intesa al tempo stesso come volta stellata e come volta celeste. I piloni del Duomo erano 52 e "simboleggiavano il ciclo dell'anno liturgico che trascorre nella perenne rievocazione della storia della Redenzione umana attraverso la liturgia delle cinquantadue settimane dell'anno". ${ }^{6}$ Il tiburio era concepito come un'allegoria del trono divino, con quattro torri agli angoli della prevista guglia:

"per fortitudine et pulchritudine tiborii vidilicet per istum exemplum in paradixo Dominus Deus sedet in medio troni, circha tronum sunt quatuor evangelistae secundum Apocalipsim"?

Il tiburio sopra l'altare (al posto della consueta crociera uguale nelle cattedrali gotiche a tutte le altre) diventava il centro simbolico, lu-

4 Cfr. J.S. Ackerman, Ars sine scientia nibil est, Gothic Theory of Architecture at the Cathedral of Milan, in The Art Bulletin, XXXI, 1949; E. Battisti. Avanguardia e conservatorismo nella storia del Duomo di Milano, in Il Duomo di Milano, Atti del Congresso..., 1969, II, pp. 45 e seg.; A. Castellano. Dal tardogotico al primo rinascimento: alcune osservazioni su progetto, disegno e cantiere, in AA.VV. Costruire in Lombardia, Milano 1983, pp. 71-72.

5 Cfr. M.L. Gatti Perer (a cura di), La dimora di Dio con gli uomini. Immagini della Gerusalemme Celeste, Milano 1983, pp.101-105.

6 Cfr. E. Cattaneo, I contenuti spirituali e religiosi nell'arte del Duomo, Milano 1980, pp.127-135.

7 Cfr. Annali, $1^{\circ}$, 19 luglio 1390 e 25 gennaio 1400 (citazione di un passo precedentemente scritto); cfr. anche M. Rossi. La correlazione di problemi statici, proporzionali e simbolici nelle prime proposte per la costruzione del tiburio del Duomo di Milano, in Arte Lombarda 58/59, 1981, p.25. 
minoso, ordinatore di tutto lo spazio interno. Era presente la numerologia: la lunghezza delle navate fino al transetto era 144 braccia (pari a 9 moduli di 16 braccia); il coro era lungo 72 braccia come il transetto (compresi le sporgenze poligonali ). I numeri 144 e 72 comparivano nelle misure della Città Celeste descritta nell'Apocalisse. (Misure identiche erano prescritte nel Capitolato del 1367 per la tribuna di S. Maria del Fiore a Firenze). Il piacentino Gabriele Stornaloco (consultato come vedremo nel 1391 per definire lo schema generale dell'alzato del Duomo) proponeva anche un complesso programma geometrico-astronomico, rappresentazione della cosmografia celeste:

"un cerchio rappresenta la volta celeste, la circonferenza rappresenta l'eclittica sulla quale i vertici di un triangolo equilatero, di un quadrato e di un esagono inscritti individuano i dodici punti zodiacali delle case e il percorso annuale del sole, della luna e dei pianeti”.

Il cerchio e il triangolo equilatero erano figure ricorrenti (insieme al quadrato) di una costruzione armonica; i dodici segni zodiacali attorno al centro del cerchio simboleggiavano gli apostoli attorno a Cristo. All'inizio del Cinquecento il Cesariano individuava nella pianta del Duomo un rombo formato da due triangoli equilateri e il cerchio come ordinatore di pianta e alzato.' (Fig. 2)

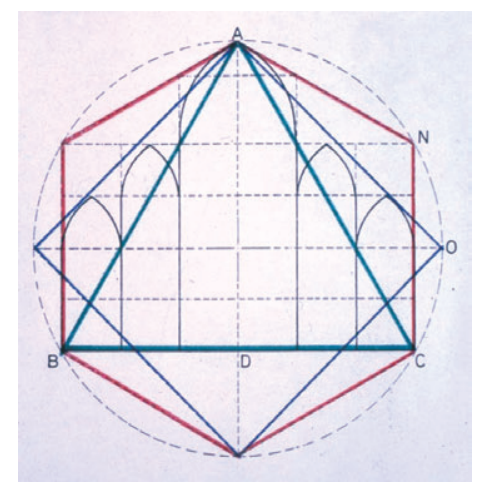

Fig. 2. G. Stornaloco, Schema geometrico per il Duomo, con triangolo, quadrato e esagono, e con le 12 case dei pianeti (1391) (da G. Valentini, 1990).

8 G. Valentini, Il Duomo di Milano. Una disputa medievale sul modello del tempio, Milano 1990, p. 56, nota 4.

9 Cfr. C. Cesariano, Vitruvii De Architectura, Como 1521, Libro Iº, foll. XIV e XV. 
Sappiamo che nel 1390 si discusse se ingrossare (e di quanto) i quattro piloni che in futuro avrebbero dovuto sostenere il tiburio, "quatuor pironi augmentantur in grossitudine...plus aliis pironis": prevalse, ma forse qualche anno più tardi, la decisione di non differenziarli dagli altri delle navate, e pertanto di aumentarli tutti, rimandando a tempi successivi e a strutture di collegamento in alto, sopra le volte, il compito di consolidare questo punto eccezionale dell'organismo. I quattro piloni ingrossati compaiono però nella pianta che l'architetto Antonio di Vincenzo (inviato a Milano nello stesso anno 1390 dalla Fabriceria di S. Petronio a Bologna) ha redatto: l'architetto annotava in proposito "va alta la cupola dal mezo braza CXIII" (oltre i 60 metri). Nel disegno della pianta Antonio di Vincenzo tracciava anche la sezione, probabilmente copiando uno degli schemi conservati nel cantiere milanese. ${ }^{10}$

Nel 1391 giunsero a Milano i tecnici tedeschi (architetti?) Hans von Fernach (o Farnech, che vi rimase fino al 1393) e Heinrich Parler di Gmund (detto Enrico di Gamondia, fino al 1392); nel 1394 giunse Hulrich Fussingen da Ulm. Due ordini di problemi furono affrontati in questi anni nel cantiere del Duomo: - la modifica parziale della pianta per migliorare l'equilibrio statico, - la decisione di stabilire la sezione, o in termini meno moderni lo schema geometrico dell'alzato, adattando il già costruito (anche a costo di demolizioni) alle necessità statiche di un nuovo modello formale. Relativamente al primo problema, proseguì a lungo una disputatio sull'opportunità di separare con muri trasversali le due navate laterali, riducendo la pianta da 5 navate a 3 navate con cappelle laterali, intramezari capellae muro. L'ipotesi difesa per esempio da Simone da Orsenigo (che morirà alla fine del 1391) ma che altri non accettavano, doveva essere già stata prevista da tempo, perché erano state costruite delle fondazioni per tali muri (ritrovate in recenti scavi). ${ }^{11}$ Alcuni proponevano non muri a tutt'altezza (come poco più tardi, nel 1396, verrà realizzato nella Certosa di Pavia) bensì una separazione solo con muri bassi, circa altitudine unius personae, teminanti in alto con un arco, volturae cum ma-

10 Il disegno, già pubblicato da L. Beltrami nel 1887, cfr. A. Cassi Ramelli (a cura di) Luca Beltrami e il Duomo di Milano, Milano 1964, p.127, è conservato nell'Archivio bolognese di S. Petronio. Anche sul verso del disegno compare la sezione di un contrafforte. La sezione di Antonio di Vincenzo del Duomo prevedeva uno sviluppo in altezza della navata centrale fino alle 84 braccia.

11 Cfr. Annali, $1^{\circ}, 20$ aprile 1390 e 2 maggio 1392. 
zoneriis. ${ }^{12}$ Anche se non a tutt'altezza, questi muri trasversali avrebbero "legato" i piloni ai contrafforti e avrebbero avuto la funzione di "controspinta", aumentando la resistenza dei piloni alle spinte verso l'esterno prodotte dalle future volte.

E' possibile che sulla prima di tali soluzioni fosse d'accordo anche Heinrich Parler, perché così erano costruite le navate laterali della cattedrale di Praga, dove lui aveva lavorato. Si dichiararono favorevoli, anni più tardi, nel 1400, anche Bernardo da Venezia e Bartolino da Novara. I due architetti di fiducia di Gian Galeazzo Visconti, avevano adottato la stessa soluzione nelle due chiese del Carmine a Pavia e a Milano, oltre che alla Certosa di Pavia ${ }^{13}$ sia per motivi statici sia per motivi tipologico-simbolici: era opportuno

"reduce la prima nave in forma de capelle cum le mezature tra l'una capella e l'altra...così vegnaravase a dare grandissima fortezza ale altre tre nave per quelli archi butanti averave più fermo el suo principio...et el corpo de la giesa parevate più bello e più con sova resdone perche el seguirava la grandezza de la Cruxe".

I due architetti suggerivano anche l'edificazione di una grande cappella in fondo al Duomo, dopo l'abside, quale mausoleo del Visconti, ma che certamente avrebbe avuto anche funzione di "controspinta", come suggerì peraltro nello stesso anno o nel seguente anche il francese Jean Mignot. ${ }^{14}$

Il dibattito più acceso, però, e certamente quello che comportava le decisioni più importanti ed eventualmente le modifiche più radicali fu quello che riguardava lo schema dell'alzato, con le scelte geometrico-proporzionali. Va precisato che non era affatto insolito nei cantieri gotici che a fronte di una pianta tracciata, e già dell'avvenuta edificazione di alcune parti, mancassero ancora le decisioni e i disegni delle sezioni verticali, le altezze dei vari piloni e le quote d'imposta delle volte: nel Duomo, non solo erano state edificate le due sagrestie laterali all'abside, ma tratti dei muri perimetrali avevano raggiunto l'altezza di 20 braccia, si lavorava nei

12 Cfr. Annali $1^{\circ}, 2$ maggio 1392.

13 Cfr. A.M. Romanini, L'architettura gotica in Lombardia, Milano 1964, p.367 e note.

14 Cfr. Annali, 1, 8 maggio 1400: lettera al duca, n.213 e anche 139. Cfr. anche G.L. Calvi. Notizie sulla vita e sulle opere dei principali architetti, scultori e pittori che fiorirono in Milano durante il governo dei Visconti e degli Sforza, Milano 1859-65, 1º , p.107. 
pressi del transetto, cruxerae, si erano confrontati modelli dei piloni, Marco da Campione ne aveva fatto un modello ligneo, ed erano stati fatti disegni pro portis et finestris ecclesiae fiendis e già si era cominciato ad eseguire alcuni motivi decorativi (archetti con fiorami) del basamento sotto i muri perimetrali e i contrafforti, primum ordinem cornisatae exterioris, scilicet folianimum (1391). ${ }^{15}$ Questo basamento (alto due braccia) con massicce modanature in serizzo, vera riedizione moderna del podio o stilobate classico, già aveva segnato la singolarità e la "diversità" del Duomo. ${ }^{16}$

Il primo a sollevare delle decise critiche a quanto era stato eseguito e a sostenere la sua inadeguatezza a "reggere la figura" corretta dell'alzato, fu Hans von Freiburg. Di fronte a una censura così preoccupante, sappiamo che fu avanzata questa richiesta:

"Che Giovanni da Firimburg ingegnere della fabbrica ponga in iscritto gli errori ed i dubbi che asserisce esservi nei lavori della fabbrica, onde vi si possa provvedere" ${ }^{17}$

Però, la volontà di provvedere doveva essere pressoché assente tra i Deputati e le maestranze, perché non si trattava di modeste modifiche e di qualche adattamento, ma di un mutamento radicale dell'impianto. Comunque, per far fronte a una così drammatica divergenza di carattere costruttivo, i Deputati chiamarono il 24 settembre del 1321 il matematicus expertus artis geometriae Gabriele Stornaloco "causa discutendi cum inzignerii... de dubiis altitudini set aliorum de quibus dubium erat inter dictis inzigneriis...". ${ }^{18}$ In un paio di mesi ${ }^{19}$ lo Stornaloco, di cui già ho ricordato il programma geometrico-astrologico, presentò un disegno d'alzato, che teneva conto della modulazione del Duomo (16 braccia), dell'altezza dei contrafforti ( 28 braccia) e della larghezza totale delle 5 navate ( 96 braccia): la sezione a cui avrebbe dovuto attenersi l'edificio nella sua crescita era definita da un triangolo equilatero, il cosiddetto schema ad triangulum, che stabiliva inoltre dei moduli quadrangolari le cui diagonali avevano la pendenza costante di 60 gradi. Lo

15 Cfr. Annali, $1^{\circ}, 16$ marzo 1390 e Appendice 1391, 45, cfr. A.M. Romanini. Op. cit., pp.364 e seg.

16 Questi argomenti sono già stati trattati in parte in L. Patetta, L'architettura del Quattrocento a Milano, Milano 1987, 2 ed. Milano 1990, pp. 35 e seg.

17 Cfr. Annali, $1^{\circ}, 12$ marzo 1391.

18 Cfr. Annali, $1^{\circ}, 24$ settembre 1391.

19 Cfr. Annali, $1^{\circ}, 26$ novembre 1391, lodi e approvazione per il lavoro dello Stornaloco. 
schema dello Stornaloco presentava una "figura" inscrivibile in un esagono e quindi in un cerchio. Tutte le quote delle navate sarebbero state precisate dallo schema, e in particolare da una serie di triangoli equilateri costruiti su ciascun modulo della pianta. La quota totale della navata centrale sarebbe stata quella dell'altezza del triangolo equilatero di base 96 braccia, cioè 84 braccia (pari a oltre 50 metri). ${ }^{20}$ (Fig. 3)

E' probabile che lo Stornaloco intendesse contrapporre il proprio schema (e forse anche un modello) a quello che doveva aver proposto Hans von Freiburg, avente di sicuro uno sviluppo maggiore in altezza, secondo gli esempi del gotico dei paesi d'Oltralpe.

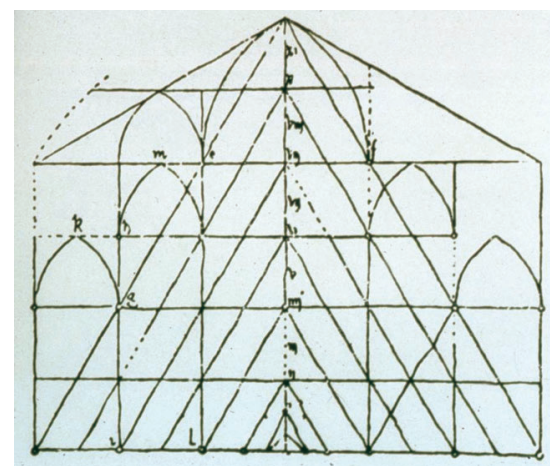

Fig. 3. G. Stornaloco, Schema generale dell'alzato del Duomo ad triangulum (1391), copia (Raccolta Bianconi, II, p.4 v.).

Lo schema dello Stornaloco finì invece per diventare un'alternativa a quello che pochi mesi più tardi proponeva l'architetto tedesco Heinrich Parler (giunto a Milano alla fine del 1391), il quale aveva esposto chiaramente, per iscritto (in 11 punti) le sue censure dei lavori e delle previsioni di lavori nel cantiere milanese. Presentato con un modello in legno (pagato l'8 febbraio 1392)21 lo schema del Parler era ad quadratum (come quello della Cattedrale di Colonia): ad una larghezza di 96 braccia delle 5 navate corrispondeva un'uguale altezza della navata centrale (92 o 96 braccia) pari a quasi 58 metri d'altezza. Ma soprattutto, lo schema del Parler non prevedeva le navate d'altezze scalari

20 Copia del disegno nel Tomo II, p. 4, della Raccolta Bianconi, presso la Biblioteca Trivulziana al Castello Sforzesco di Milano.

21 Cfr. Annali, 1ㅇ, 2 febbraio e 8 febbraio e 25 febbraio 1392. 
"a gradonata", come di consueto nel gotico italiano, ma prevedeva le due navate laterali della stessa altezza (come nelle ballenkirche) alte 48 braccia, cioè più basse della centrale di ben 48 braccia (quasi 26 metri). Sarebbe stato possibile, naturalmente, realizzare il tipico claristorio e aprire una serie di grandi finestre. Ma era evidente a tutti che una tale sezione del Duomo avrebbe reso necessaria la costruzione di archi rampanti, dei quali le maestranze lombarde non avevano alcuna esperienza. Forse i maestri lombardi avrebbero avuto anche qualche difficoltà a realizzare macchine di sollevamento dei pesi richieste da un organismo di altezza così inconsueta. Per queste ragioni, e forse anche per qualche preoccupazione d'ordine economico, i Deputati della Fabbrica del Duomo indissero un'assemblea per analizzare gli 11 punti della critica dell'architetto tedesco, nonché il suo modello. Parteciparono alla $d i$ sputatio molti maestri italiani, tra cui Lorenzo degli Spazi, Bernardo da Venezia, Giovannino de' Grassi, Stefano Magatto e l'ingegnere Giovanni da Ferrara (allora molto stimato). E' probabile che nell'occasione sia stato esaminato, e oggetto di confronto, il modello della soluzione ad triangulum dello Stornaloco, non a caso fatto eseguire poco prima del 23 febbraio 1392 dai carpentieri Simone da Cavagnera e Simone da Piacenza sul disegno di Gabriele Stornaloco, probabilmente interpretato e sviluppato con ulteriori approfondimenti. ${ }^{22}$

Le decisioni assembleari del primo maggio 1392 furono contrarie a Heinrich Parler (che infatti fu licenziato e lasciò Milano) e confutarono punto per punto i suoi dubbi.

Non solo confermarono l'impianto generale del Duomo, ma deliberarono che "le strutture hanno solidità sufficiente a sostenere carichi anche maggiori" (punti 1 e 2); "che la chiesa possa salire fino alla figura triangolare e non oltre" (punto 3), che i piloni con basi e capitelli debbano salire a quaranta braccia e non oltre" (punto 4), "che non si debba tramezzare con un muro le cappelle della chiesa" (punto 7); "che non debba eseguire un corridoio (claristorio) nella nave maggiore" (punto 8), "che non si modifichi nulla dei piloni e contrafforti eseguiti" (punti 9 e 10); "che i piloni minori debbono essere alti ventotto braccia e i piloni mediani di ulteriori dodici braccia” (punto 11). Unica concessione

22 Cfr. Annali, $1^{\circ}, 19$ ottobre 1391 e 23 febbraio 1392; cfr. per le ipotesi sugli schemi dello Stornaloco e per la pubblicazione integrale in latino e italiano degli 11 punti discussi nell'assemblea della Fabbrica, G. Valentini, op. cit. pp.61-83, il quale indica per lo Stornaloco il nome originario di Scovaloca, della sua famiglia piacentina. 
fu di far costruire i piloni delle navate dello stesso diametro dei quattro piloni sotto il futuro tiburio (che già erano stati ingrossati). (Fig. 4)

Nei mesi successivi, forse con consigli di Giovannino de' Grassi (attivo al Duomo fino al 1398, anno della morte e autore dei singolari capitelli alti 10 braccia cioè sei metri e con nicchie occupate da statue) fu deciso di fare un altro modello, che correggeva prudentemente lo schema dello Stornaloco, che pure era stato adottato dall' assemblea dei Deputati. Anche se alcuni dubbi dovevano serpeggiare tra i maestri attivi nel cantiere, tanto che fu consultato ancora un forestiero nel 1394, Ulrich Fussingen (e alcuni difetti furono infatti corretti in questi anni), ${ }^{23}$ si stabilì che a tale modello dovesse attenersi chiunque fosse in futuro incaricato di dirigere i lavori al Duomo:

"ut exemplum remaneat semper ad evidentiam cuiuslibet personae pro avvisamento operum ipsius fabricae, cum contigat ipsam finire et cooperire...". ${ }^{24}$

L'altezza totale della navata centrale fu ridotta a 76 braccia (invece che alle 84 braccia previste dallo schema dello Stornaloco) sovrapponendo dopo la quota dei muri perimetrali (braccia 28) lo schema del triangolo egizio, un triangolo rettangolo con i lati nel rapporto 3:4:5, che di quello equilatero era meno pendente. Non sappiamo se tale decisione fu presa in seguito a un nuovo dibattito assembleare con argomenti teorici, oppure dai maestri del cantiere nel corso dei lavori, con prassi empirica e con intuizioni, come scopriremo più avanti. Certamente la decisione fece sì che il Duomo avesse una così modesta differenza di altezza tra le navate da poter avere una copertura a terrazzi e archi rampanti con un ruolo staticamente secondario. E' però interessante notare che la scelta di adottare contemporaneamente due schemi, quello del triangolo equilatero (con pendenza di 60 gradi) in basso e quello del triangolo egizio (con pendenza 37 gradi) in alto, trova oggi la sua legittimazione nella moderna Tecnica delle Costruzioni, che tollera spinte laterali più accentuate nelle parti alte degli edifici, dove l'entità dei pesi è minore, mentre prescrive una convergenza più prossima al

23 Cfr. C. Boito, Il Duomo di Milano e i disegni per la sua facciata, Milano 1889, p. 120; sugli errori in parte corretti in parte restati nella struttura cfr. C. Ferrari da Passano, i restauri del Duomo in Le origini lombarde del Duomo di Milano, AA.VV. Il Duomo di Milano, Milano 1973 e La Fabbrica e il suo tempo, Milano 1985.

24 Il passo è riportato negli Annali in data 28 dicembre 1399, ma si riferisce a sette anni prima, cfr. L. Beltrami, 1887, cit. p.21 e cfr. L. Patetta. Op. cit., p.36 e note. 
"nocciolo" delle strutture verticali nelle parti basse, dove i carichi sono ovviamente molto maggiori.

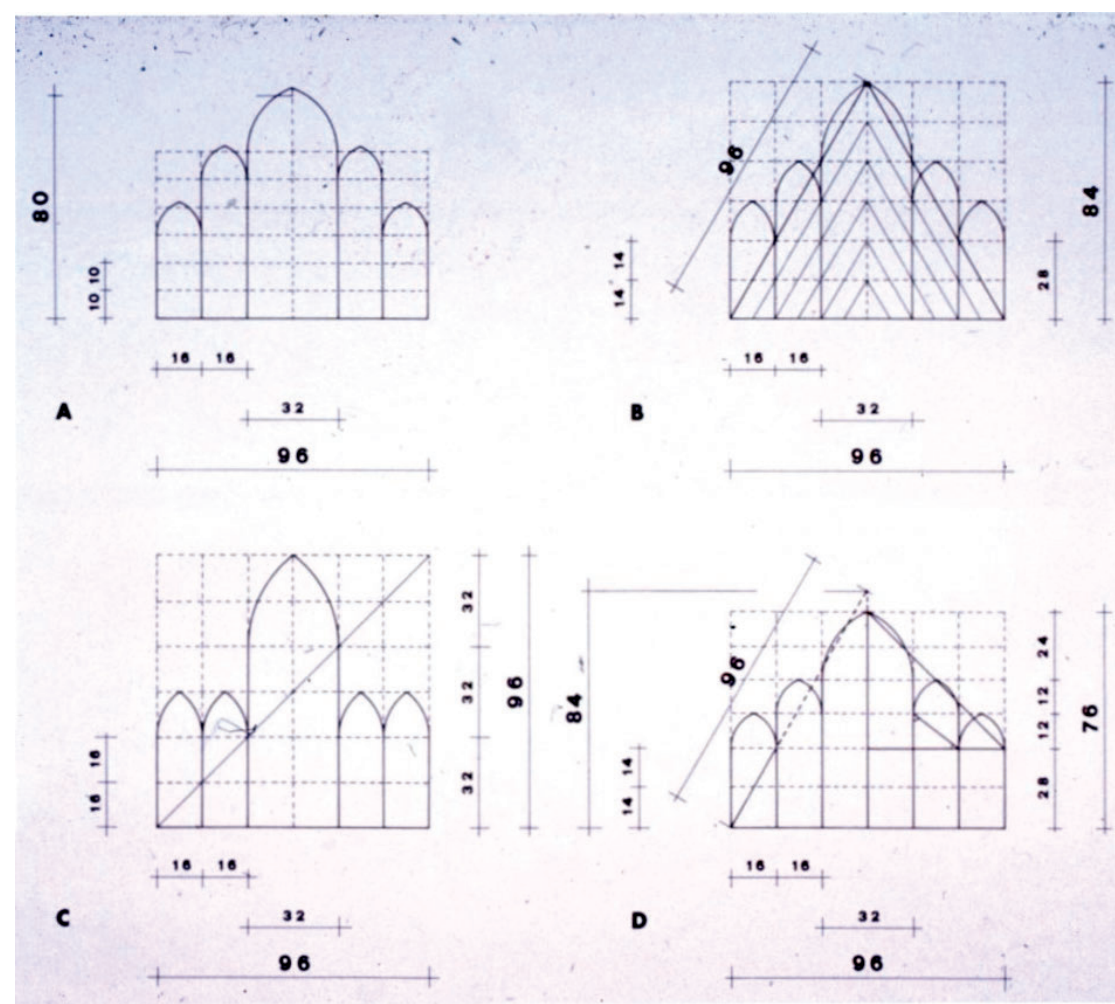

Fig. 4. Schemi generali dell'alzato del Duomo: A) Antonio di Vincenzo; B) Gabriele Stornaloco; C) H. Parler, ad quadratum 1392; D) schema adottato dalla Fabbrica nel 1392 (da L. Patetta, 1987).

Un'altra intuizione può essere segnalata: la scelta, forse dettata anche da un'esigenza di risparmio, di costruire i piloni con la tecnica della struttura "a camicia", cioé con un anello di marmo di Candoglia e all'interno una colata di calcestruzzo di malta, sabbia, ghiaia e anche cocci di laterizio (con opportuni "legamenti"). Per la prima volta nel 1882, contraddicendo quanto riteneva Viollet le Duc, questi piloni criticati dai tecnici forestieri risultavano più resistenti di quelli costruiti interamente in pietra (quindi molto più pesanti). Si scoprì dunque tra la fine dell'Ottocento e i primi del Novecento, che le strutture 
"a sacco" per la loro "discontinuità" erano più vantaggiose delle strutture "omogenee". ${ }^{25}$ (Fig. 5)

Dal 1399 al 1401 fu presente nel cantiere il francese Jean Mignot, che fece disegni per piloni e finestre nella zona absidale. L'architetto Mignot criticò con 54 proposizioni ${ }^{26}$ sia la decisione di abbandonare il riferimento rigoroso ad un'unica "figura" adottando schemi "compositi" e quindi eretici rispetto ai modelli, sia l'esecuzione di alcune parti:

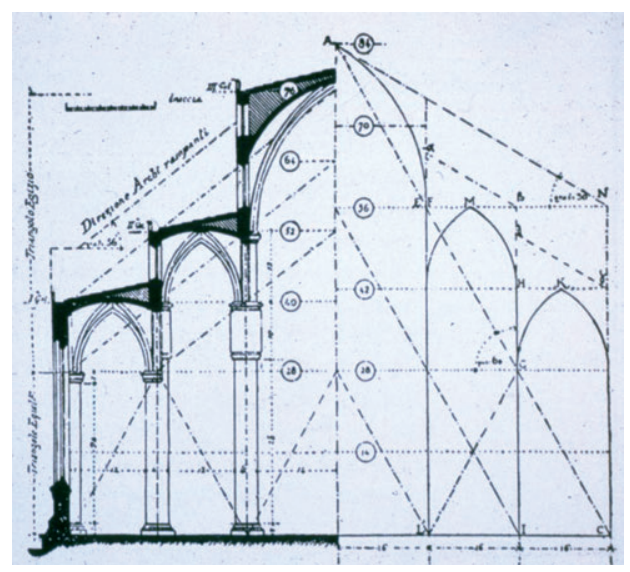

Fig. 5. Sezione del Duomo: a sinistra l'inclinazione degli archi rampanti della realizzazione; a destra lo schema dello Stornaloco, 1391 (disegno di L. Beltrami, in A. Cassi Ramelli, 1964).

per esempio, osservò che la parte absidale non era fortis sicut debet, tanto da fargli suggerire l'edificazione di una cappella retrostante con funzione di controspinta statica. ${ }^{27}$ Egli espresse un parere radicalmente negativo sulla previsione di costruire un tiburio circondato da quattro torri, perché avrebbe provocato il crollo di tutte le strutture circostanti e accusò i maestri locali di non voler mai cambiare indirizzo ma al contrario "volunt aut per eorum lucro aut timore...conse-

25 Questa questione costruttiva fu sollevata la prima volta da Anatole De Baudot in occasione del restauro della cattedrale di Nantes, cfr. Parigi, Archives Nationales, F 197773,20 aprile 1882.

26 Cfr. J. Ackerman, Op. cit. pp.84 e seg.

27 Cfr. Annali, $1^{\circ}, 13$ aprile 1399 (prima presenza documentata di Jean Mignot a Milano), 1 maggio 1400, 8 maggio 1400 e poi 20 novembre 1401 . 
gui fabricam super defectibus". ${ }^{28}$ Per il Mignot, se non si fosse cambiato tutto il programma costruttivo la rovina sarebbe stata totale: in sintesi, il crollo: Dicta fabbrica ruet pro certo. Discussioni drammatiche e ferocemente polemiche si tennero tra il maggio e il novembre 1401. Gli ingegneri e i maestri del Duomo controbbatterono una per una tutte le accuse e le critiche del Mignot: sostennero, per esempio, che pur non corrispondendo a schemi "canonici" gli archi ogivali delle navate, e in particolare quelli sotto il tiburio, non provocavano incontrollabili spinte laterali, perché gli sforzi erano sempre ricondotti ai piloni (non dant impulum contrafortibus); dichiararono che la fabbrica era ben salda in ogni sua parte; che nelle strutture erano stati introdotti tiranti in ferro (per esempio tra piloni e contrafforti); che $i$ blocchi di Serizzo erano ben ancorati ("bene inclavati") che alcune parti come le grandi finestre erano già state collaudate e approvate dallo stesso architetto francese, perché bene inclavatae cum bonis ferris implombantis. I lombardi lo accusarono di non saper tener conto delle grandi differenze tra il Serizzo e il Candoglia del Duomo e i marmi di Francia, dichiarando inoltre che tutti gli accorgimenti adottati (tiranti, catene, chiavi metalliche ecc.) erano non dei "ripieghi" ma elementi dedotti da una lunga pratica costruttiva. (Fig. 6)

Dalle polemiche non restarono esclusi temi prettamente formali, come quello dei capitelli che il Mignot avrebbe voluto di altezza quattro volte le basi dei piloni, mentre erano molto più alti e "abnormi" cioè $\mathrm{I} / 5$ del fusto dei piloni anziché $\mathrm{I} / 8$ e quindi fuori dalle proporzioni vitruviane (!).

Riguardo agli schemi geometrici dell'alzato generale della fabbrica, i lombardi, premesso che la scelta del triangolo era suffragata addirittura da Aristotele, ${ }^{29}$ conclusero capovolgendo la famosa affermazione perentoria di Jean Mignot Ars (cioè la pratica) sine scientia (senza l'insieme delle regole geometrico-prooporzionali) nibil est, nel senso opposto: Scientia sine arte nibil est. Cioè, i maestri del Duomo replicarono che lo "scientismo" medievale basato su numeri e forme geometriche non poteva condizionare in senso assoluto gli elementi della costruzione: Scientia geometriae non debet in iis locum habere et pura scientia est unum et ars est aliud. Senza una verifica nell'applicazione

28 Cfr. Annali, $1^{\circ}, 25$ maggio 1400.

29 Cfr. P. Frankl. The secret of Mediaeval Masons, in The Art Bulletin, XX, 1945, p. 58 e The Gothic Literary Sources and Interpretations, Princeton 1960, p. 79. 
pratica, la scienza cui si appellava l'architetto francese restava dunque una pura astrazione, e non diventava "scienza architettonica".

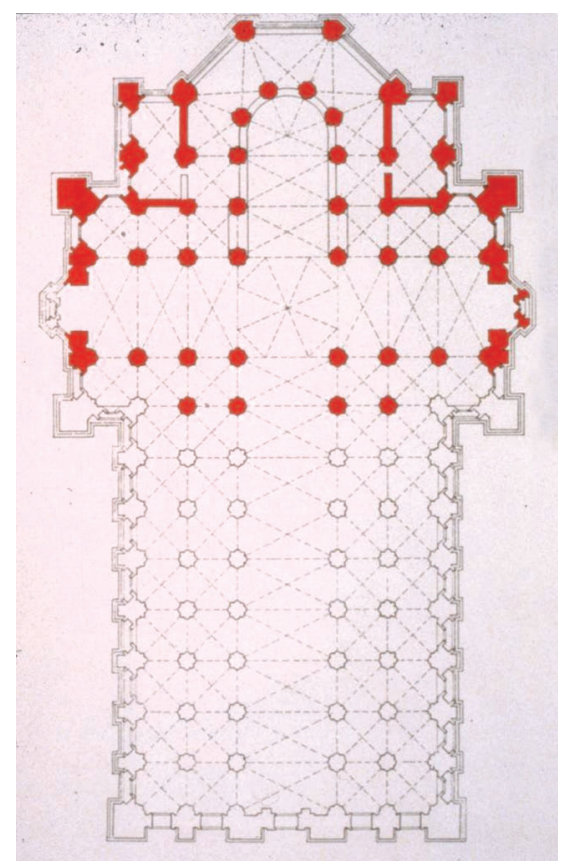

Fig. 6. Le strutture del Duomo edificate all'inizio del Quattrocento.

Sono evidenti i significati e le conclusioni che si possono trarre da queste dispute. Gli architetti forestieri, che provenivano dai cantieri di alcune delle cattedrali più famose d'Europa (dove però le grandi soluzioni strutturali e formali erano state prese nei secoli passati e dove essi attendevano per lo più ai completamenti tardogotici) non seppero dare alcuna risposta puntuale ai problemi che il Duomo poneva. Non fu dunque, come spesso si è scritto, lo scontro tra il "mestiere" di semplici costruttori, la "praticaccia" (come la chiamerà cinquant'anni più tardi il Filarete) e la scienza del gotico, perché questi architetti tedeschi e francesi non erano né in possesso di una grande esperienza pratica né portatori di reali competenze scientifiche. Sembravano piuttosto presentare alcune formule erudite già di tipo accademico, più che frutto di importanti esperienze personali. Pertanto essi delusero, perché non seppero partecipare a una sperimentazione complessa di tecniche e di 
materiali mai prima di allora impiegati in dimensioni così impegnative (strutture in Serizzo, marmo di Candoglia, mattoni, conglomerato di malta e scaglie di laterizio e pietre ecc.). Essi non vollero valutare le differenze dei materiali e adattare le soluzioni "canoniche" a necessità differenti e risposero con schemi prestabiliti e regole rigide alle questioni poste da una tecnologia processuale, con modifiche e adattamenti, ${ }^{30}$ forse inesperta e arrischiata, ma vivacemente creativa (come lo era stato il gotico duecento anni prima). Gli architetti forestieri censurarono severamente, in nome di un purismo strutturalista, i tiranti in ferro, che in parte erano una scelta economica (compensando, sostituendo e diminuendo la pietra con la loro capacità di "trazione") in parte erano forse provvisori, in attesa che i pesi successivi (per esempio delle guglie) equilibrassero definitivamente l'organismo architettonico.

Valutando negativamente i "pentimenti" e gli esperimenti tipici dei cantieri delle cattedrali e il cambiamento di qualche elemento costruttivo, ${ }^{31}$ nonché rifiutando di accettare scelte formali che non appartenevano alla tradizione del gotico d'Oltralpe, (come il basamento "classico" di cui si è detto, dei muri contraffortati, del tiburio ecc.) si rese infruttuoso il confronto e le disputationes della tradizione dei grandi cantieri. E si dimostrò che la grande epoca dei "costruttori di cattedrali" era definitivamente finita. Le resistenze dei responsabili della fabbrica del Duomo fecero sì che si evitasse di assumere posizioni antistoriche. (In disaccordo con quanto io scrivo, ricorrono in molti studi storici sul Duomo riconoscimenti della validità delle critiche pronunciate sulle sue strutture, e la convinzione di trovarsi di fronte a "provincialismo", a "soluzioni spesso di retroguardia, e contradditorie", a "incapacità tecnica" e a "sbandamento stilistico"). ${ }^{32}$

Non a caso il cantiere del Duomo, la "gran macchina" che secondo Giorgio Vasari tanto aveva colpito il Bramante "che considerata

30 Interessanti le osservazioni in tal senso di M. Manieri Elia in Storia e uso dei modelli architettonici, Bari 1982, p.144.

31 Come provano, per esempio, le tracce e i resti di archi e volte in laterizio esistenti nella sagrestia nord.

32 Numerose le opinioni opposte a queste mie. Tra le altre, per esempio, quelle di J.S. Ackerman, cit. 1949, pp.85-87; E. Battisti, cit. 1969, II,p.45-52; A.M. Romanini. Il Duomo di Milano, Milano 1973, I, pp.101-102; e A. Castellano, cit. 1983, pp. 71-72.

33 Cfr. G. Vasari, Le Vite dei più eccellenti pittori, scultori e architetti, edizione Roma 1991, p.583. 
questa fabbrica si risolvé del tutto a darsi all'architettura" ${ }^{33}$ costituì per i maggiori architetti del Rinascimento a Milano il laboratorio più stimolante, dove affrontare in un senso nuovo i rapporti tra forma e struttura, tra scienza e tecnica, tra teoria e pratica e tra tradizione e rinnovamento. $^{34}$

34 Mi riferisco alla presenza geniale di Guiniforte Solari, al problema del tiburio affrontato anche in senso teorico da Leonardo, Bramante e Francesco di Giorgio, agli interventi dell'Amadeo, agli studi del Cesariano, ai progetti di Tibaldi, ecc. 